\title{
Author Correction: Pericyte-like spreading by disseminated cancer cells activates YAP and MRTF for metastatic colonization
}

Ekrem Emrah Er, Manuel Valiente D, Karuna Ganesh, Yilong Zou, Saloni Agrawal, Jing Hu, Bailey Griscom,

Marc Rosenblum, Adrienne Boire DD, Edi Brogi, Filippo G. Giancotti, Melitta Schachner, Srinivas Malladi and Joan Massagué

Correction to: Nature Cell Biology https://doi.org/10.1038/s41556-018-0138-8, published online 23 July 2018.

In the version of this Article originally published, the authors inadvertently included the term 'pericytic mimicry' in relation to ref. 54. This has now been corrected by inserting an additional reference at position 51 and amending the text in the Discussion relating to 'pericytic mimicry', ref. 54 and pericyte-like spreading. The original refs 51-70 have also been renumbered. Furthermore, Fig. 81 has been amended to remove the term 'pericyte mimicry' that the authors had included inadvertently during figure preparation. These corrections have been made in the online versions of the Article.

\section{Author Correction: CRISPR-Cas9-mediated base-editing screening in mice identifies DND1 amino acids that are critical for primordial germ cell development}

Qing Li, Yanjing Li, Suming Yang, Shuo Huang, Meng Yan, Yifu Ding, Wei Tang, Xiwen Lou, Qi Yin, Zhanfei Sun, Lei Lu, Huijuan Shi, Hongyan Wang, Yong Chen (D) and Jinsong Li (D)

Correction to: Nature Cell Biology https://doi.org/10.1038/s41556-018-0202-4, published online 1 October 2018.

In Fig. 2a of this Technical Report originally published, the authors inadvertently used the same set of images for the 4B2N1 and 4B2N3 cells when preparing the figure. The three images (bright field, Oct4-EGFP and pCAG-mRFP) of 4B2N3 cells have now been replaced with the correct versions. The source data for the four cell lines in Fig. 2a, captured in the three independent experiments, have been deposited to Figshare (https://doi.org/10.6084/m9.figshare.7387607.v1), and the figure legends and Methods section have been amended to reflect this. Additionally, the unprocessed blots in Supplementary Fig. 7 corresponding to the top right 'WCL IB: Flag' panel of Fig. 7e were mistakenly duplicates of the unprocessed blots for the bottom left 'IP Flag IB: HA' panel of Fig. 7e, and all unprocessed blots for Supplementary Fig. 6 were mislabelled as blots corresponding to Supplementary Fig. 7. Supplementary Fig. 7 has now been updated to show the correct unprocessed blots for the bottom left 'IP Flag IB: HA' panel of Fig. 7e and to correct the labelling of the unprocessed blots corresponding to Supplementary Fig. 6.
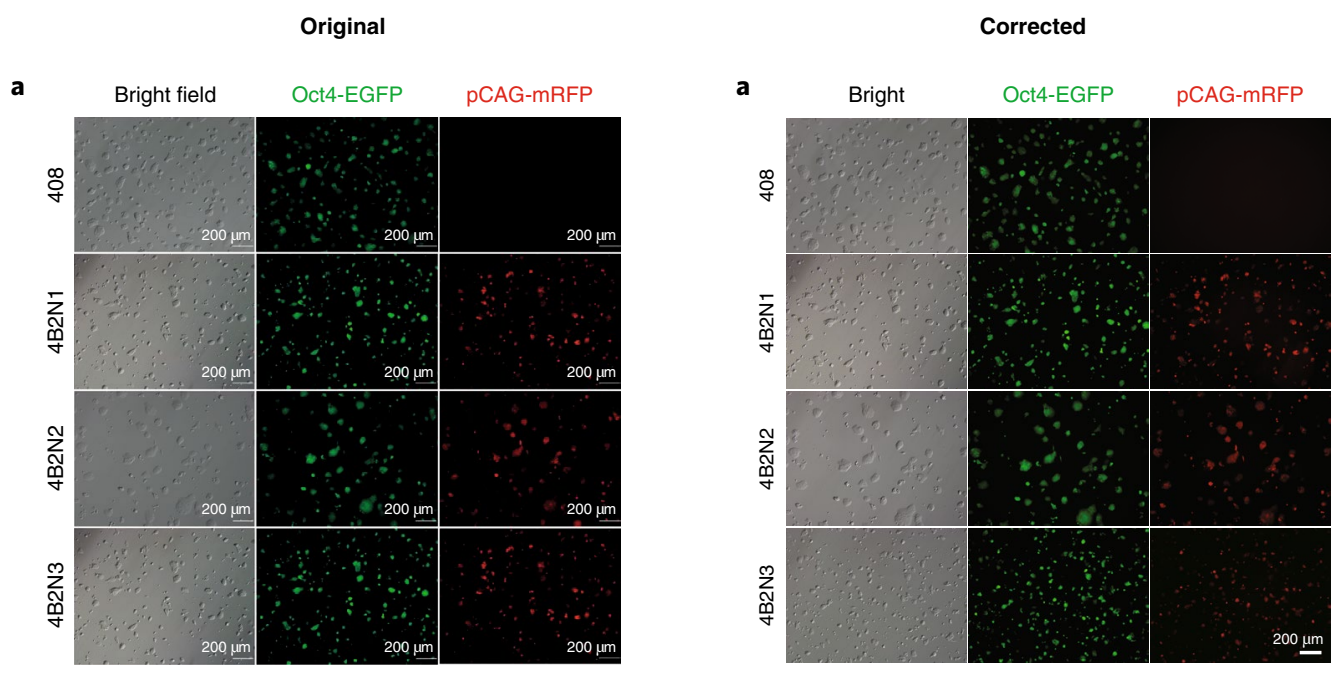

Fig. 2 | Orginal and Corrected. 